American Journal of Applied Sciences 4 (5): 317-322, 2007

ISSN 1546-9239

(C) 2007 Science Publications

\title{
Resource Leveling Using Petrinet and Memetic Approach
}

\author{
K. Raja and S. Kumanan \\ Department of Production Engineering \\ National Institute of Technology Tiruchirappalli, India
}

\begin{abstract}
The problem of scheduling activities dynamic for a project, within the available resources and adhering to precedence relationships is NP hard combinatorial. Traditional approaches and their extensions are limited in application for simultaneous scheduling of resources with leveling. Considering the need for improved modeling and scheduling techniques, this paper proposes the use of Petrinet based approach aided by memetic algorithm. Manufacturing projects involving either a single or multi resources can be optimally leveled and scheduled. The effectiveness of this approach is demonstrated with case studies.
\end{abstract}

Key words: Project management, Petrinet, memetic algorithm, resource leveling

\section{INTRODUCTION}

Project management is the process of managing, allocating and timing resources in order to achieve a given objective in an expedient manner. Leveling of resources has been getting attention from researchers. Resource leveling procedures are aimed to obtain the even usage of resources and to avoid high peak or very low resource requirements. The basic steps of resource leveling programs are as follows; 1) Create a resource profile based on the early start time of each activity using traditional computations, 2) Shift non- critical activities to achieve uniform resource levels of resources. In order to execute the second step, various techniques are usually adopted. These resource-leveling problems are combinatorial nondeterministic polynomial time complete problems. Though in the small project situation resources can be acquired or released in any desired amounts, maintaining use of different resources at a constant rate is very important in large-scale projects with multiple resources. Many researches have focused on single resource leveling procedures. Burgess and Kilkerew $^{[1]}$, Kumar and Rajendran ${ }^{[2]}$ proposed heuristic approaches. Easa ${ }^{[3]}$ and Bandelloni et al. ${ }^{[4]}$ have recommended the mathematical approaches. Younis and $\mathrm{Saad}^{[5]}$ developed mathematical model for optimal resource leveling of multi resource projects. The optimization approaches for resource leveling utilizes enumeration method and hence for a large problem turns out as a difficult combinatorial problem. The heuristic approaches do not conduct exhaustive search and its outcome varies from problem to problems ${ }^{[6]}$.

This paper address the use of Petrinet based software PERTIPM in modeling projects requiring single or multi resources and proposes the use of memetic algorithm search for optimal resource leveled scheduling of a project.

Modeling project with Petrinet: A new project management model with ability to define features accurately and encourage efficient communications between all involved in the planning and execution of the project will yield positive results. Petrinet is a graphical and mathematical modeling tool that has been successfully applied extensively in the areas of performance evaluation, communication protocol, legal system and decision making models Agerwala ${ }^{[7]}$ and Murata $^{[8]}$. Project management has also been identified ${ }^{[9]}$ as a prospective area, where the modeling power of Petrinet can be used Dynamic representation and online monitoring of activities and resources are very helpful throughout the phases of management. Many researchers have attempted Petrinet as a management tool. Magott (1989) proposed a combined generalized stochastic Petri nets and Pert networks for the performance evaluation of concurrent process. Pagnoni ${ }^{[10]}$ detailed Petrinet and its use as a planning language for projects. Lee et al.(1994) developed a model called PM-net for representing and monitoring the software development process is presented This model provides information for progress management, as well as information of project status at different levels of detail, for the benefit of project managers. Kim et al. ${ }^{[11]}$ introduced an interactive provision for regenerating and re scheduling of activities. Meherez et $a l^{[12]}$ reviewed the properties of petrinet models with view of management perspective as certain management decision problems are not often easily modeled using traditional decision making

Corresponding Author: $\quad$ K. Raja, Research Scholar, National Institute of Technology. Tamilnadu, India 
frameworks and as management decision problems may possess characteristics that are difficult of describe fully with any single decision making frame work such as decision tree or a network model. Kusumoto et al. ${ }^{[13]}$ proposed new model with GSPNs for software projects and an estimation method for the quality, cost and delivery date. Shih and Leung (1997) proposed a management Petrinet but with multiple kinds of places and transitions. Kumar and Ganesh $^{[14]}$ describe the use of petrinet to facilitate resource allocation in projects under some conditions commonly encountered in practice. To make petrinet to suit certain applications certain modifications and extensions are to be done. A consistent framework for modeling and analysis of characteristics exhibited by the projects such as precedence relations, sequential execution, conflict, concurrency, synchronization, merging, selecting alternatives, dynamism, etc needs attention.

Software: Petrinet-aided software, PETRIPM is developed to deal with project planning with stochastic activity times. It has been completely designed using Visual Basic, so as to make use of its graphical and customized application development tools. The package is developed as menu driven with user-friendly message displays. The software has main menu with Project planning module as sub menu. The planning phase is usually identified with construction of the model graphically, during which specific decisions are made on the method of performing jobs as well as their technological ordering and simultaneously, stochastic times can be assigned. The project planning module has been developed to deal with project modelling and analysis. Extensions to Petri nets are required considering the intricacies of project management ${ }^{[12]}$. In a Petri net:

A place represents the start or completion of an activity. Circles represent them. A transition represents activity, which is a time /resource consuming part of a project. Bars represent them.

An arc connects places and transitions. It is not allowed to connect two places or two transitions. The arcs are either from a transition to a place or from a place to transition. Token represent resources of the operation. These are represented by black dot. A marking is an assignment of tokens to the places of a Petri net. If a place is marked with ' $k$ ', we say that place is marked with ' $\mathrm{k}$ ' tokens. Pictorially, we place ' $\mathrm{k}$ ' black dots (tokens) in place. A marking is denoted by $\mathrm{M}$, an $\mathrm{m}$-vector where $\mathrm{M}$ is total number places. The $\mathrm{p}$ component of the $\mathrm{M}$, denoted by $\mathrm{M}(\mathrm{p})$, is the number of tokens in place $P$.

\section{Problem definition}

Single resource leveling: The objective is to schedule project with minimum resource fluctuations. Assumptions are as follows:

A project consists of a number of activities. Resource required per unit time for each activity is known and constant throughout its execution. Activity cannot start until its entire predecessor has been completed.

No activity pre-emption is allowed. Resource is unlimited

Multi resource leveling: The objective is to schedule project with minimal resource fluctuations simultaneously for all resources. A project consists of number of activities, which use specified types of resources. An Activity cannot start until all its predecessors have been completed. Activity preemption is not allowed. Resources are unlimited.

Proposed solution methodology: Evolutionary algorithms (EAs) are a class of search and optimization techniques that work on a principle inspired by nature: Darwinian Evolution. The concept of natural selection is captured in EAs. Specifically, solutions to a given problem are codified in so- called chromosomes. The evolution of chromosomes due to the action of crossover, mutation and natural selection are simulated through computer code. It is now well established that pure EAs are not well suited to fine tuning search in complex combinorial spaces and that hybridization with other techniques can greatly improve the efficiency of search ${ }^{[15]}$. The combination of EAs with local search (LS) was named "memetic algorithms" (MAs) in [P. Moscato].The operation of EAs start with random generation of initial population and then the selection, crossover, local search mutation and local search are produced until the best population is found .The proposed memetic algorithm flowchart shows in the figure 1

Memetic parameters: A search for an optimum set of early start dates for the non critical activities is made through GA and Local search method that is called as Memetic algorithm. The set of parameters for Memetic search module are detailed in the following sections.

Coding of the string: In this application, string or chromosome corresponds to the set of start dates for the non-critical activities. The length of the chromosome is equal to the number of non-critical activities. For example, the string length is equal to 4 
elements as there are 4 non critical activities. The first element indicates that activity 3 to be started on day 6 and second element indicates that the activity 4 to be started on day 3 and so on. The coding of string depicted in the figure 2

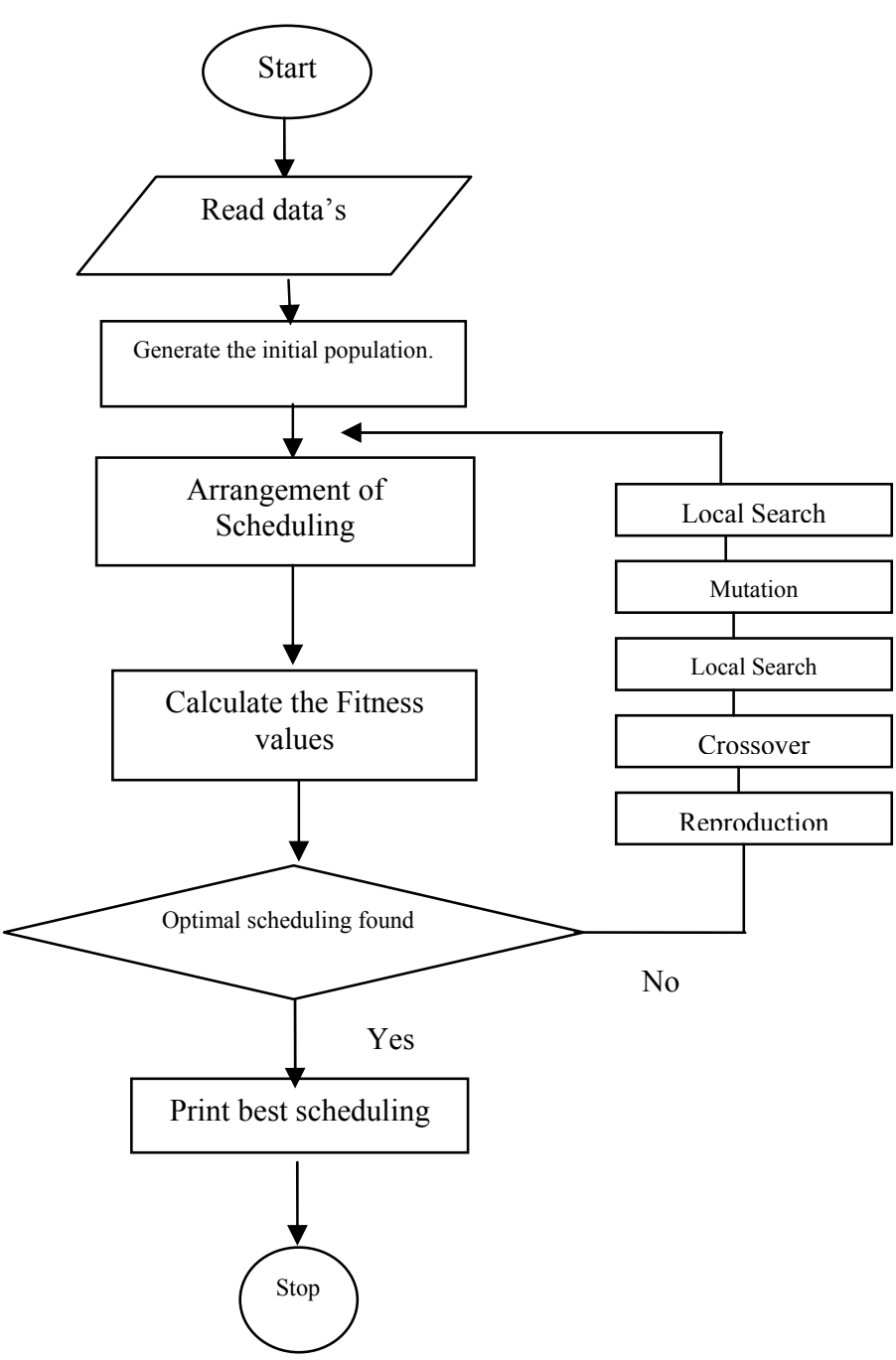

Figure 1Flow chart of the proposed memetic algorithm

Initial population: A user defined initial population is set and is generated randomly. In the present case, the population size is set at 20 (Gold berg 1992).

\begin{tabular}{|l|l|l|l|l|}
\hline chromosome & 6 & 3 & 11 & 12 \\
\hline
\end{tabular}

\begin{tabular}{|l|l|l|l|l|}
\hline Non critical set & 3 & 4 & 6 & 7 \\
\hline
\end{tabular}

Figure.2: coding of a string for single resource leveling
Objective function: The objective function is to minimize the square sum of daily resources of project activities (critical as well as non critical activities).It is written as

$$
\mathrm{Z}=\sum_{\mathrm{i}=1}^{\mathrm{T}} \mathrm{Ri}^{2}
$$

Where $\mathrm{Ri}=\sum_{\mathrm{j}=1}^{\mathrm{N}} \mathrm{r}_{\mathrm{ji}} ; \quad \mathrm{N}=$ number of enabled transitions. And $\mathrm{r}_{\mathrm{ji}}=$ resource required by $\mathrm{j}^{\text {th }}$ enabled transition in $\mathrm{i}^{\text {th }}$ period.

Evaluative/Fitness function: Since it is a minimization problem, the objective function value itself is used as a fitness function. Between two chromosomes, if the objective function value of the first is less than second then first one is selected.

Selection for Reproduction: Strings with higher fitness value are selected for crossover and mutation using stochastic sampling with the probability of selecting each evaluated as

$p_{s i}=\frac{1 / f_{i}}{\sum_{i=0}^{q} 1 / f_{i}}$

Where $f_{i}$ is the fitness value of the $i^{\text {th }}$ string and $\mathrm{i}=0,1,2 \ldots . \mathrm{q}$.

Crossover: This is the next operation in the memetic approach. It partially exchanges information between the two selected strings. In the present approach, each string in the reproduction population is subjected to crossover operation with a specified probability of crossover. The probability of crossover will be changed by a adaptive method. Once the string is chosen for crossover, its mate and crossover site is selected randomly. Crossover for single resource leveling shown in the figure 3

\begin{tabular}{|c|c|c|c|c|}
\hline Parent 1 & 2 & 4 & 12 & 10 \\
\hline Parent 2 & 3 & 6 & 8 & 9 \\
\hline Off spring & 2 & 4 & 8 & 9 \\
\hline Off spring & 3 & 6 & 12 & 10 \\
\hline
\end{tabular}

Figure 3 Crossover for Single resource leveling 
Local search for improving off spring: Improving the $\sum \mathrm{Ri}^{2}$ value (minimizing) using local search technique for offspring 1 and 2 .

\section{Pseudo of a local search algorithm}

Begin

Set best $=i$, Set iteration $=0$

Repeat until (pivot rule is satisfied) do

Generate the next neighbor $(\mathrm{j})$

Calculate $\sum \mathrm{Ri}^{2}=\mathrm{f}(\mathrm{j})$.

If $f(j)$ is better than $f$ (best) then

Set best $=\mathrm{j}$, OD $\quad$ Set $\mathrm{i}=$ best

end

Only optimized offspring are added to the initial parent population. However, in the case of genetic

irrespective of offspring value $\left(\sum \mathrm{Ri}^{2}\right)$ (high or low) are added to the initial parent population.

Mutation: This is the process of randomly modifying the string with a probability. This helps to perfect the list of some potentially useful string and avoids a solution sticking the local optimum. The element is the random choice for mutation and replace with one of the value at random from the list of possible start dates of activity. After the mutation is completed, strings and are improved by local search technique by choosing the elements. Mutation process depicted in the figure 4

\begin{tabular}{|l|l|l|l|l|}
\hline Parent & 3 & 6 & 8 & 9 \\
\hline Activity Set & 3 & 4 & 6 & 7 \\
\hline
\end{tabular}

\begin{tabular}{|l|l|l|l|l|}
\hline Off spring & 3 & 6 & 10 & 9 \\
\hline
\end{tabular}

Figure 4 Mutation process in single resource leveling

Local search: Improving the objective function value (minimizing) using local search technique for offspring 3 and 4 .The procedure adopted for multi resource leveling is similar to the procedure discussed above except for the variation of objective function, which is detailed below the objective function is to minimize the sum of absolute deviations of each of the resource desirable and actual resource (for critical as well as non critical activities) .Sum of the absolute deviations for each resource, throughout the project duration as computed as:

Minimize $Z(r)=\sum_{i=1}^{r} \operatorname{Abs}(e(i, r)) \times W$

where $e(i, r)=y(i, r)-c(r)$.e $(i, r) \quad$ is the error between the desirable level $\mathrm{c}(\mathrm{r})$ and the actual resource level, $y(i, r)$ at each time unit for each resource $\mathrm{r}$. W is the resource weight factor.

\section{RESULTS AND DISCUSSION}

The single resource problem in Kartam and Tongthong is used for validating the proposed method. Figure 5shows the modeling of project activity network with PERTIPM software and Table 2 shows the precedence relations, resource requirements of the project. A memetic algorithm has been used to level the resources and results Fig. 1a and Table 1 shows early schedule of the project, Fig. $1 \mathrm{~b}$ and Table 3 shows resource leveling chart of proposed memetic algorithm method and Fig. 1c and Table 4 shows resource leveling chart of proposed alternate memetic algorithm method. Kumar and Rajendran obtained improved solution compared to Burgess and Killebrew and Jeetendra and chetty obtained good solution than all others by own heuristic method. The peak requirement of resources is found to be reduced from 14 to 11 resulting in an improvement of 7.8percentage than heuristic method developed by Jeentendra and Chetty. The measure of effectiveness resulted in an improvement of 7 percentages.

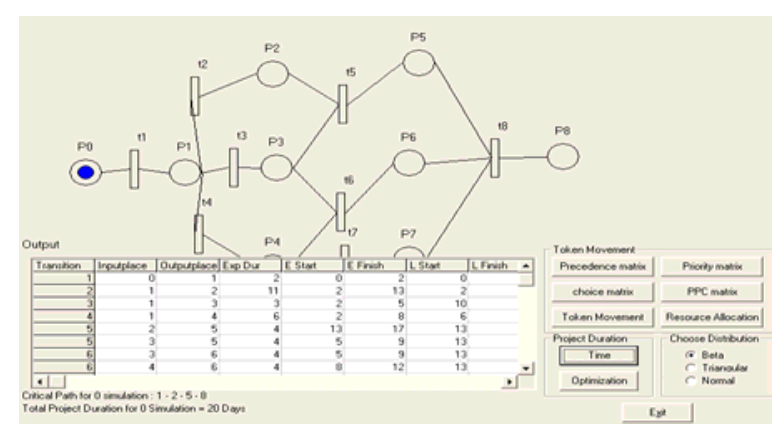

Fig. 5: Project Network PETRI-PM software module 
Table 1: Early schedule of the project ${ }^{[6]} \sum \mathrm{Ri}^{2}=1239$

\begin{tabular}{|c|c|c|c|c|c|c|c|c|c|c|c|c|c|c|c|c|c|c|c|c|}
\hline Activity & 1 & 2 & 3 & 4 & 5 & 6 & 7 & 8 & 9 & 10 & 11 & 12 & 13 & 14 & 15 & 16 & 17 & 18 & 19 & 20 \\
\hline 1 & & \multirow{7}{*}{0} & \multirow{7}{*}{$\begin{array}{l}3 \\
4\end{array}$} & & \multirow{7}{*}{$\begin{array}{l}3 \\
4\end{array}$} & & & & & & & \multirow{4}{*}{3} & \multirow{4}{*}{3} & & & & & & & \\
\hline 2 & \multirow{6}{*}{0} & & & \multirow{6}{*}{$\begin{array}{l}3 \\
4\end{array}$} & & & & & & & & & & & & & & & & \\
\hline 3 & & & & & & & & & & & & & & & & & & & & \\
\hline 4 & & & & & & 3 & 3 & 3 & 3 & 3 & 3 & & & & & & & & & \\
\hline 5 & & & & & & & & & & & & & & 0 & 0 & 0 & & & & \\
\hline 6 & & & & & & 5 & 5 & 5 & 5 & 5 & 5 & & & 6 & 6 & 6 & 0 & & & \\
\hline $\begin{array}{l}7 \\
8\end{array}$ & & & & & & & & & & & & 3 & 3 & 3 & 3 & 3 & 6 & 1 & 1 & 1 \\
\hline $\mathrm{R}_{\mathrm{i}}$ & 0 & 0 & 7 & 7 & 7 & 8 & 8 & 8 & 8 & 8 & 8 & 6 & 6 & 9 & 9 & 9 & 6 & 1 & 1 & 1 \\
\hline
\end{tabular}

Table 2: Project network input data ${ }^{[6]}$

\begin{tabular}{|c|c|c|c|c|c|}
\hline Transitions & $\begin{array}{l}\text { Resource } \\
\text { Units/day }\end{array}$ & A & M & B & $\begin{array}{l}\text { Immediate } \\
\text { Predecessors }\end{array}$ \\
\hline 1 & 0 & 1 & 2 & 3 & \\
\hline 2 & 3 & 10 & 11 & 12 & 1 \\
\hline 3 & 4 & 2 & 3 & 4 & 1 \\
\hline 4 & 5 & 5 & 6 & 7 & 1 \\
\hline 5 & 0 & 3 & 4 & 5 & 2,3 \\
\hline 6 & 6 & 3 & 4 & 5 & 3,4 \\
\hline 7 & 3 & 4 & 5 & 6 & 4 \\
\hline 8 & 1 & 2 & 3 & 4 & $5,6,7$ \\
\hline
\end{tabular}

Table 3: Optimal schedule of the project by the proposed $\operatorname{method} \sum \mathrm{Ri}^{2}=885$

\begin{tabular}{|c|c|c|c|c|c|c|c|c|c|c|c|c|c|c|c|c|c|c|c|c|}
\hline Activity & 1 & 2 & 3 & 4 & 5 & 6 & 7 & 8 & 9 & 10 & 11 & 12 & 13 & 14 & 15 & 16 & 17 & 18 & 19 & 20 \\
\hline 1 & & & & & & & & & & & & & & & & & & & & \\
\hline 2 & & & & & & & & & & & & 3 & 2 & & & & & & & \\
\hline 3 & & & & & & & & & & & & & 3 & & & & & & & \\
\hline 4 & 0 & 0 & 3 & 3 & 3 & 3 & 3 & 3 & 3 & 3 & 3 & & & & & & & & & \\
\hline 5 & & & 5 & 5 & 5 & 5 & 5 & 5 & 4 & 4 & 4 & & & 0 & 0 & 0 & & & & \\
\hline $\begin{array}{l}6 \\
7\end{array}$ & & & 5 & 5 & 5 & 5 & 5 & 5 & & & & 3 & & 6 & 6 & 6 & 0 & & & \\
\hline 8 & & & & & & & & & & & & 3 & 3 & 3 & 3 & 3 & 0 & 1 & 1 & 1 \\
\hline & 0 & 0 & 8 & 8 & 8 & 8 & 8 & 8 & 7 & 7 & 7 & 6 & 6 & 9 & 9 & 9 & 6 & 1 & 1 & 1 \\
\hline
\end{tabular}

Table 4: Optimal schedule (Alternate solution) of the project by the proposed method $\sum \mathrm{Ri}^{2}=885$

\begin{tabular}{|c|c|c|c|c|c|c|c|c|c|c|c|c|c|c|c|c|c|c|c|c|}
\hline Activity & 1 & 2 & 3 & 4 & 5 & 6 & 7 & 8 & 9 & 10 & 11 & 12 & 13 & 14 & 15 & 16 & 17 & 18 & 19 & 20 \\
\hline 1 & \multirow{6}{*}{0} & \multirow{6}{*}{0} & \multirow{6}{*}{$\begin{array}{l}3 \\
4 \\
5\end{array}$} & & & & & & & & & & & \multirow{6}{*}{0} & & \multirow{6}{*}{0} & \multirow{6}{*}{0} & & & \\
\hline 2 & & & & & & & & & 3 & 3 & 3 & 3 & 3 & & & & & & & \\
\hline $\begin{array}{l}3 \\
4\end{array}$ & & & & 3 & 3 & 3 & 3 & 3 & & & & & & & & & & & & \\
\hline 5 & & & & 4 & 4 & & & & & & & & & & & & & & & \\
\hline 6 & & & & 5 & 5 & 5 & 5 & 5 & 6 & 6 & 6 & 6 & & & 0 & & & & & \\
\hline $\begin{array}{l}7 \\
8\end{array}$ & & & & & & & & & 3 & 3 & 3 & 3 & 3 & & & & & 1 & 1 & 1 \\
\hline $\mathrm{R}_{\mathrm{i}}$ & 0 & 0 & 12 & 12 & 12 & 8 & 8 & 8 & 12 & 12 & 12 & 12 & 6 & 0 & 0 & 0 & 0 & 1 & 1 & 1 \\
\hline
\end{tabular}




\section{CONCLUSION}

Resource leveling is an important phase of project scheduling. Cost effective project schedules are obtained by reducing resource fluctuations over the duration of a project. Considering the advantages of modeling power of Petrinet and optimization efficiency of memetic algorithms, a method of modeling a project with petrinet and optimal scheduling by memetic search method is proposed in this paper. Resource leveling algorithms for single level resource are described. Method for local search technique for the crossover and the mutation in single resource leveling procedures makes the algorithm generic and self directive. The procedure is validated with case studies from literature. The proposed methods not only can handle real time changes of the project but also creates competent alternate (it is compared with previous algorithms) resource leveled schedules that provide flexibility to the user.

(A)

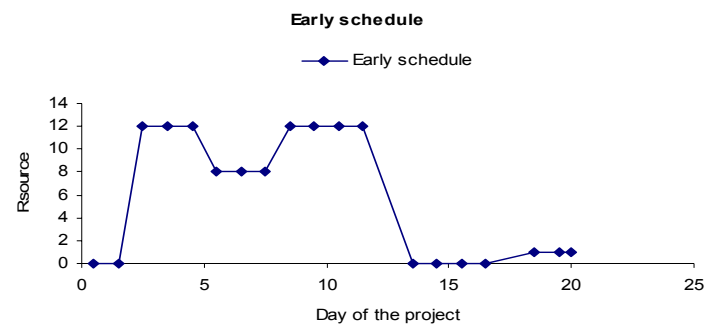

B)

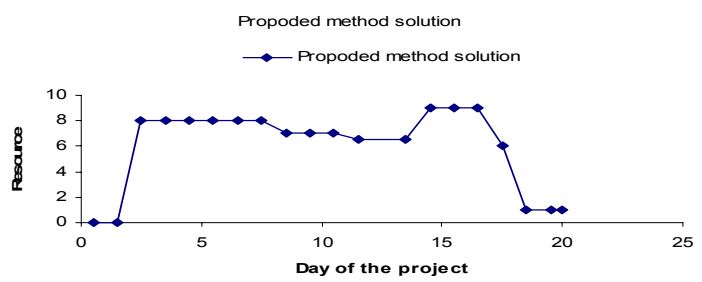

(C)

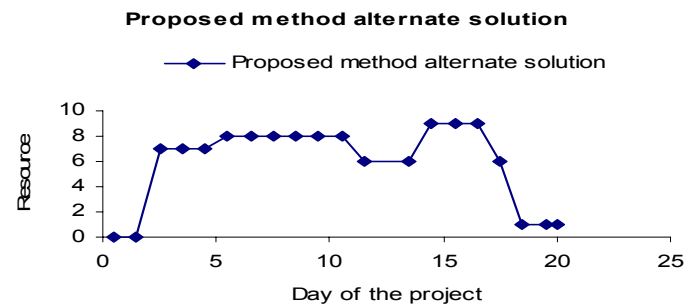

Fig. 4: Resource leveling chart for A) Early scheduling of the project ( Kartam and Torgthong), B) Proposed method solution using memetic approach, C) Proposed method alternate solution using memetic approach

\section{REFERENCES}

1. Burgess, A.R. and J.R. Kilebrew, 1962. Variation in activity level on a cyclical arrow diagram. J. Indust. Engg.,13: 76-83.

2. Kumar, V.K.A. and C.R. Rajendran, 1993. Man power resource leveling in the maintenance of the drill shop computer simulation approach. Intl. J. Model. Simulation, 13: 152-155.

3. Essa, S., 1989. Resource constrained project scheduling. Manag. Sci., pp: 944-955.

4. Bandelloni, M., M. Tucci and R. Rinaldi, 1994. Optimal resource leveling using non serial dynamic programming. Eur. J. Oper. Res., 78: 162-177.

5. Younis, M.A. and B. Sadd, 1996. Optimal resource leveling of Multi resource projects. Computers and Industrial Engineering.

6. Kartam, N. and T. Tongthong, 1997. Potential of Artificial Intelligence for engineering .Design Analysis and Manufacturing ,11:171-185.

7. Agerwala, T., 1979. Putting Petri Nets to work. IEEE Computer, 12: 85-94.

8. Murata, T., 1989. Petrinet Properties, analysis and applications. Proc. IEEE, 77: 541-580.

9. Peterson, J.L., 1981. Petrinet Theory and Modeling of Systems. Prentice Hall, Inc. N.J.

10. Pagnoni, A., 1990. Project Engineering Computer Oriented Planning and Operational Decision Making. Berlin springer-Verlag.

11. Kim, J., A.A. Desrochers and A.C. Sanderson, 1995. Task planning and project management using Petrinet. Proc. First Intl. Symp. Assembly and Taskplanning, pp: 265-271, Pittsburgh IEEE.

12. Meherez, A., M. Muzumdar, W. Acar and G. Weinroth, 1995. Petrnet model view of decision making. An Operational Management Analysis Omega, 23: 63-78.

13. Kusumoto, S., O. Mizuno, T. Kikuno, Y. Hirayama, Y. Takagi and K. Sakamoto, 1997. A new software project simulator based on generalized stochastic Petrnets. Proc. $19^{\text {th }}$ Intl. Conf. on Software Engg., 17-23 May, Boston, MA, pp: 295-308.

14. Kumar, V.K.A. and L.S. Ganesh, 1998. Use of Petrinet for resource allocation in projects. IEEE Trans. on Engg. Manag., 45: 49-56.

15. Goldberg, D. and S. Voessner, 1999. Optimizing global-Local search hybrids. Inproc. Genetic Evol. Comput. Conf., W. Banzhaf, J. Daida, A. Eiden, M.Garzon,V. Honavar, M. Jakaiela and R. Smith, Eds., pp: 220-228. 